\title{
The fission yield calculations with Langevin model, Hauser-Feshbach statis- tical decay, and beta decay
}

\author{
Shin Okumura ${ }^{1, *}$, Toshihiko Kawano ${ }^{2, * *}$, and Satoshi $\mathrm{Chiba}^{3, * * *}$ \\ ${ }^{1}$ NAPC-Nuclear Data Section, International Atomic Energy Agency \\ ${ }^{2}$ Los Alamos National Laboratory \\ ${ }^{3}$ Laboratory for Advanced Nuclear Energy, Tokyo Institute of Technology
}

\begin{abstract}
We performed the calculations of de-excitation of the primary fission fragments by the HauserFeshbach statistical decay followed by the $\beta$ decay of de-excited fission products. We used the primary fission fragment mass distributions $Y_{P}(A)$, total kinetic energy $\operatorname{TKE}(A)$, and its width $\sigma_{T K E}(A)$ as input, which were calculated with the Langevin model using macroscopic-microscopic models of the potential energy surface. The prompt neutron multiplicity $\bar{v}$ and the independent fission product yield (FPY) $Y_{I}(Z, A, M)$ and cumulative FPY $Y_{C}(Z, A, M)$ are calculated by the Hauser-Feshbach statistical decay and $\beta$ decay calculations, respectively. The calculated $\bar{v}$ was overestimated approximately $17 \%$ compared to the evaluated data. The decay heats from $\beta$ and $\gamma$ were in accordance with the experimental results. The $\beta$ delayed neutrons yieild was also overestimated.
\end{abstract}

\section{Introduction}

The fission product yield (FPY) has been of great interest for fundamental understanding of the nuclear fission process. There are still major gaps in our understanding of origins of the FPY distribution by any theories or models. The nuclear fission begins with the formation of compound nucleus with a projectile. The compound nucleus changes its shape towards the evolution on the potentialenergy surface defined by shape parameters describing its deformation until it splits into two fragments. The primary fission fragments formed just after scission are usually excited. These fragments de-excite by emitting prompt photons and neutrons to reach their ground states or isomeric states. The de-excited fragments, also called fission products, undergo $\beta$ decay towards $\beta$ stability, which produces the decay heat and $\beta$ delayed neutrons. Since these stages of the fission process comprise different physics, no reliable theory has yet been developed that can interpret all fission observables consistent with the primary fission fragment distributions.

The most of fission observables during the fission process are defined by characteristics of the primary fission fragment, which are still not well known. The fission fragment can be characterised by its yield as a function of mass $A$, charge $Z$, isomeric state $M$, total excitation energy (TXE), and spin $J$ and parity $\Pi$, i.e., $Y_{P}(Z, A, M$, TXE, $J, \Pi)$. Such information cannot be obtained experimentally due to their very short half-lives compared to the experimental timescale, therefore numerous attempts in theoretical descriptions of the nuclear fis-

\footnotetext{
*e-mail: s.okumura@iaea.org

**e-mail: kawano@lanl.gov

***e-mail: chiba.satoshi@nr.titech.ac.jp
}

sion process have been made. For example, some of fission fragment characteristics have been reproduced based on the microscopic theory [1], Monte Carlo techniques [2, 3], Brownian shape motion [4], random walk [5], or Langevin equations [6-8]. The de-excitation models, which treat sequential emissions of photons and neutrons from the primary fission fragment, have been developed by adopting Weisskopf spectrum [2, 9, 10], the HauserFeshbach statistical decay theory [3, 11-14] or by the event-by-event analysis [10]. The $\beta$-decay observables have been calculated by the summation of $\beta$-decay chain of the fission products [15]. Since the experimental data for the primary fission fragment are not so well known, a comparison of the calculated data with the experimental data requires any decay models, i.e. de-excitation and $\beta$ decay, where large amounts of experimental data are accessible.

In this work, we combined the primary fission fragment mass yield $Y_{P}(A)$, mass averaged total kinetic energy $\langle\mathrm{TKE}\rangle(A)$ and its width $\sigma_{\mathrm{TKE}}(A)$ calculated by the four dimensional Langevin model using the macroscopicmicroscopic potential-energy surface $[7,8]$ with the deexcitation model based on the Hauser-Feshbach statistical decay theory $[16,17]$ followed by the $\beta$ decay calculation [18] using the decay data library. Using theoretical calculated fission fragment distributions is attractive for some actinides which are difficult to handle experimentally and for various incident neutron energies which are difficult to generate the monoenergetic neutron source. This combined approach will allow us to estimate fission observables of experimentally unknown areas. We exemplify the model calculation for ${ }^{235} \mathrm{U}\left(\mathrm{n}_{t h}, f\right)$ and compare the calculated and experimental independent FPY $Y_{I}(Z, A, M)$, neu- 


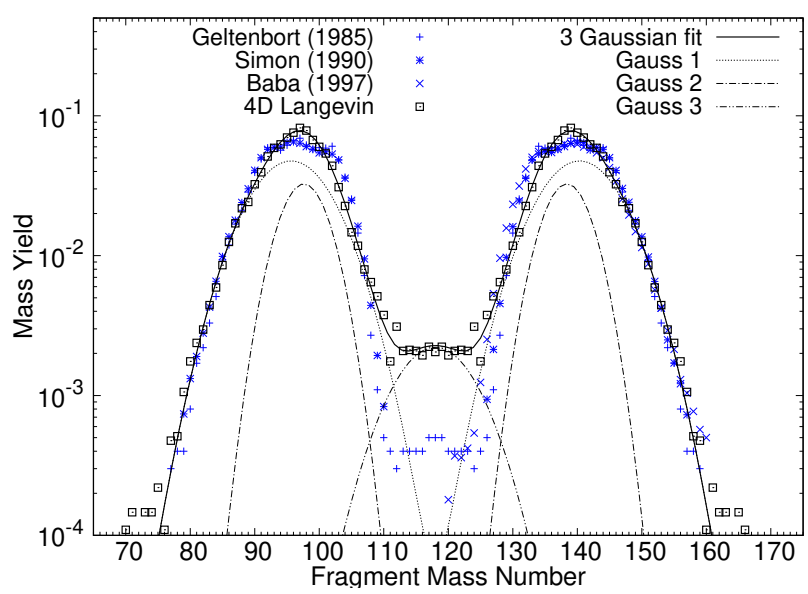

Figure 1. The three Gaussian fitted primary fission product mass yield compared with experimental data. The open squares are Langevin calculated primary fission product mass yield $Y_{P}(A)$ taken from Ref. [7] and solid line is the result of the three Gaussian fitting.

tron multiplicity $\bar{v}$, cumulative FPY $Y_{C}(Z, A, M)$, decay heat, and delayed neutron yield $v_{d}$.

\section{Models}

\subsection{Primary fission fragment generation}

The complete description of the four dimensional Langevin model and calculation parameters used here can be found in Refs. [7, 8]. The Langevin model simulates the Brownian shape-motion on the macroscopic-microscopic description of the potential-energy surface. The calculation gives mass asymmetry, total kinetic energy (TKE), and nuclear temperature, etc. for each fission event based on shapes of separated fragments at the scission. From the mass asymmetry and the compound nucleus mass, one can obtain fragment masses of the fission fragment pair. Note that here we treat the binary fission only. Since this study focuses on demonstrating the method, we utilized the fission event data which have the largest number of events, because it is important to parameterize the distribution with the good statistics. After accumulating more than $10^{5}$ fission events, the mass averaged fission fragment yield $Y(A)$ was generated and normalized the total yield to $200 \%$. For TKE, the fragment-mass averaged TKE $\langle\mathrm{TKE}\rangle(A)$ and its width (the standard deviation) at given mass $\sigma_{\mathrm{TKE}}(\mathrm{A})$ can be obtained by averaging over fragment mass. We note that the excitation energy considered in this work can be treated as the thermal energy.

We fitted the $Y(A)$ with three Gauss functions. As seen from Fig. 1, the calculated primary fission fragment $Y(A)$ shows the differences in the symmetric region $(110 \leq A \leq$ $130)$ and the peak region $(A \sim 95$ and $A \sim 140)$ compared to the experimental data for ${ }^{235} \mathrm{U}\left(\mathrm{n}_{\mathrm{th}}, f\right)$ reaction. Since the charge of the fragment is not know from Langevin model calculation, we utilize the $Z_{P}$ model [19] to generate $Y(Z, A)$. The $\langle\operatorname{TKE}\rangle(A)$ was fitted by a simple ana-

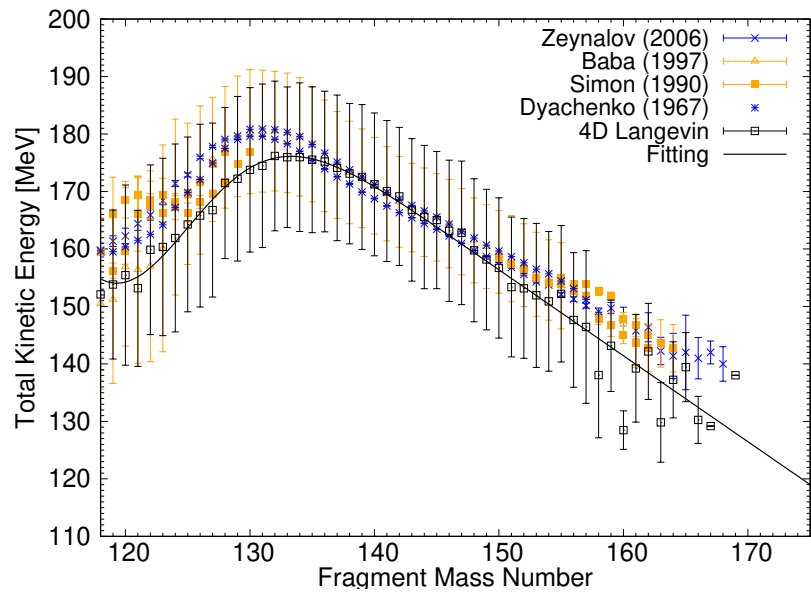

Figure 2. The fitted total kinetic energy as a function of fission fragment mass $\langle\mathrm{TKE}\rangle(A)$ taken from Ref. [7] compared with experimental data. The open squares are Langevin calculated $\langle\mathrm{TKE}\rangle(A)$ and solid line is the fitting curve.

lytical formula. These fitting parameters were used for the the Hauser-Feshbach calculation.

\subsection{De-excitation of primary fission fragment}

The fragment de-excitation process was calculated using the $\mathrm{HF}^{3} \mathrm{D}$ code [17] which treats the fission fragmet as a compound nucleus based on the Hauser-Feshbach statistical decay theory $[13,17]$. For the Hauser-Feshbach calculation, it additionally requires the excitation energy and its distribution for all primary fragments. The total excitation energy (TXE) of a certain fission fragment pair can be calculated from the energy balance of the reaction. Energy sharing between the complementary fission fragments using the anisothermal parameter used in this study are described elsewhere [3,17, 20-22]. The initial spin $J$ and parity $\Pi$ distribution of the fission fragments are also required as input, as the Hauser-Feshbach decay conserves the angular momentum. We assumed that the $J$ follows the Gaussian type of function and the $\Pi$ follows equal probability of positive and negative. The details are described in Ref. [17]. In this way, we generated the necessary fission fragment distributions $Y_{P}(Z, A, M$, TXE, $J, \Pi)$ deterministically and integrate all over them. After the de-excitation of fission fragments was simulated, one can obtain independent FPY $Y_{I}(Z, A, M)$.

\subsection{Beta decay of fission product}

The cumulative FPY $Y_{C}(Z, A, M)$ can be calculated from $Y_{I}(Z, A, M)$ by tracking the $\beta$-decay chain for each fission product, or it can be calculated directly in the equilibrium time domain [23]. The decay scheme, half-life, branching ratio, and $\beta, \gamma$, and total decay energy released per decay of each unstable nuclide were imported from the ENDF/B-VII decay data library. The summation calculation was employed to predict aggregate properties of the fission products such as decay heat. The decay heat and 


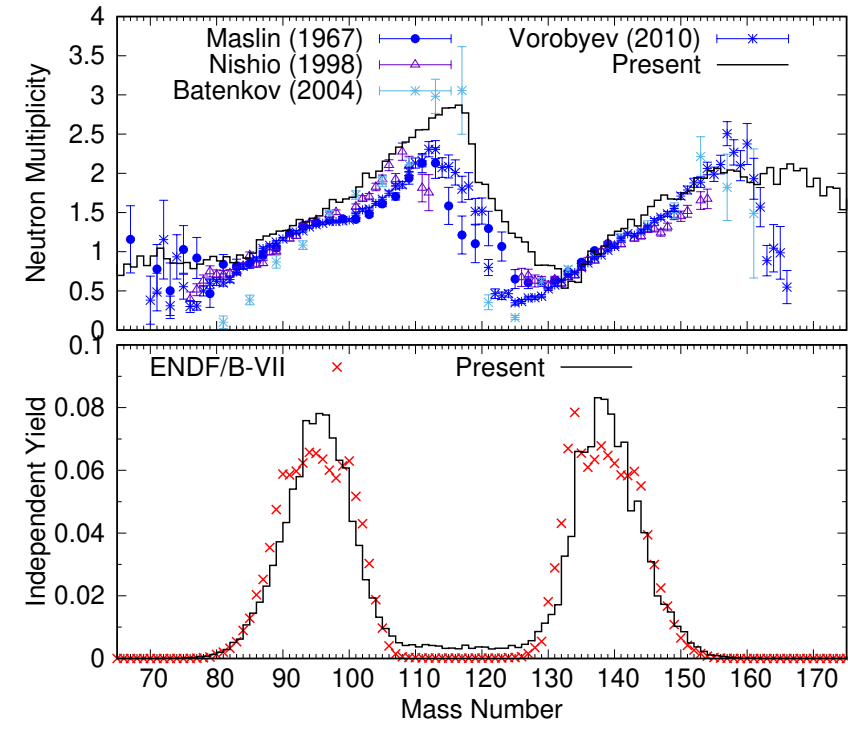

Figure 3. The calculated neuron multiplicity and the independent FPY as a function of mass

the delayed neutron are calculated by the sum of the $\beta$ and $\gamma$ energies and neutron released from all individual $\beta$-decay nuclides [15].

\section{Results}

In our previous study, the de-excitation calculation has been done using the primary fission fragment that is generated by the $Y(A)$ and $\operatorname{TKE}(A)$ fitted to experimental data, together with the $Z_{P}$ model $[17,18,24]$. In this work, we explore the feasibility of utilizing of fission fragment distributions generated by the fission model described in Sec. 2.1 and Refs. [7, 8]. We conducted the HauserFeshbach de-excitation calculations by employing the fitting parameters obtained from the calculated $Y(A)$ and $\operatorname{TKE}(A)$ for ${ }^{235} \mathrm{U}\left(\mathrm{n}_{\mathrm{th}}, f\right)$ from Ref. [7] into $\mathrm{HF}^{3} \mathrm{D}$ code [17].

An important feature of $\langle\mathrm{TKE}\rangle(A)$ shown in Fig. 2 is that, the larger $\langle\mathrm{TKE}\rangle$ will result a lower $\langle\mathrm{TXE}\rangle$, which leads the less prompt neutron and photon emissions. From the lower $\langle\mathrm{TKE}\rangle$ around the symmetric region $(118 \leq$ $A \leq 130$ for heavy fragment), one can predict the higher prompt neutron and photon emissions from fission fragments in this mass region. In addition, the multiplicities of prompt neutron and photon emissions also strongly depend on the primary fission fragment $Y(Z, A)$. From $Y(A)$ shown in Fig. 1, the symmetric region has larger yield than the experimental data, and this also will enhance the multiplicities of prompt particle emissions.

From the de-excitation calculation, the average prompt neutron multiplicity $\bar{v}$ was 2.84 neutrons / fission, that is slightly higher than the evaluated value 2.41 neutrons/fission in ENDF/B-VII. The top panel of Fig. 3 shows the calculated neuron multiplicity as a function of fragment mass $\bar{v}(A)$ compared with some experimental data. The calculation reproduced the sawtooth structure of $\bar{v}(A)$, but overestimated the wide ranges of masses around
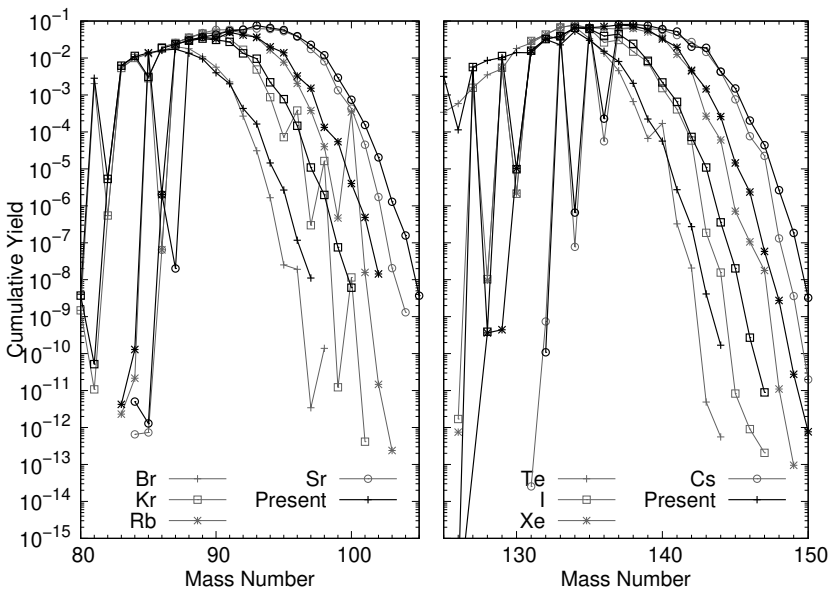

Figure 4. The calculated cumulative FPY for selected elemtens from light (left panel) and heavy (right panel) fragment. In this figure, only ground state yields are shown (metastable states are exculded).

the symmetric region $(105 \leq A \leq 130)$ as predicted. The changes in the primary mass yields and $\langle\mathrm{TKE}\rangle(A)$ shown in Fig. 1 and 2, respectively, can be interpret to a change in prompt fission observables. Thecalculated independent FPY as a function of mass $Y_{I}(A)$ is shown in the bottom panel of Fig. 3 compared with the FPY data in ENDF/BVII. As a result of the prompt neutron emissions, fine structures of the independent FPY appeared around peak reagion $(A \sim 95$ and $A \sim 140)$, but they were not reproduced perfectly. Such differences are attributed to the the primary fission product yield used as input of the deexcitation calculation shown in Fig. 1. The overall discrepancy are larger than that of our previous study [17], nevertheless, we examined the effect on the $\beta$-decay observables from using the calculated $Y_{I}(Z, A, M)$.

The calculated $Y_{I}(Z, A, M)$ was used as input of the $\beta$ decay and summation calculations. Figure 4 shows the calculated cumulative FPY $Y_{C}(Z, A, M)$ of some selected elements from light and heavy fragments around peak regions compared with the evaluated FPY data taken from ENDF/B-VII. Although there are discrepancies between our calculation and ENDF/B-VII, the width of the mass distribution in particular elements and the orders of magnitude down to considerably low yields as $10^{-10}$ were reproduced.

Figure 5 shows the calculated decay heat by the summation calculation method using the $Y_{I}(Z, A, M)$. The energy release from $\beta$ showed a good agreement with experimental data while that from $\gamma$ was underestimated in the early time $(\leq 100 \mathrm{~s})$ from fission burst. The total decay heat reflects lower decay heat from $\gamma$. The delayed neutron yield $v_{d}$ calculated from the summation method yields 0.01623 which is a few \% larger than that of the evaluated data (0.01585 in both ENDF/B-VII and JENDL4.0).

\section{Conclusion}

We have used the theoretically calculated primary fission fragment mass and TKE distributions as input of the 


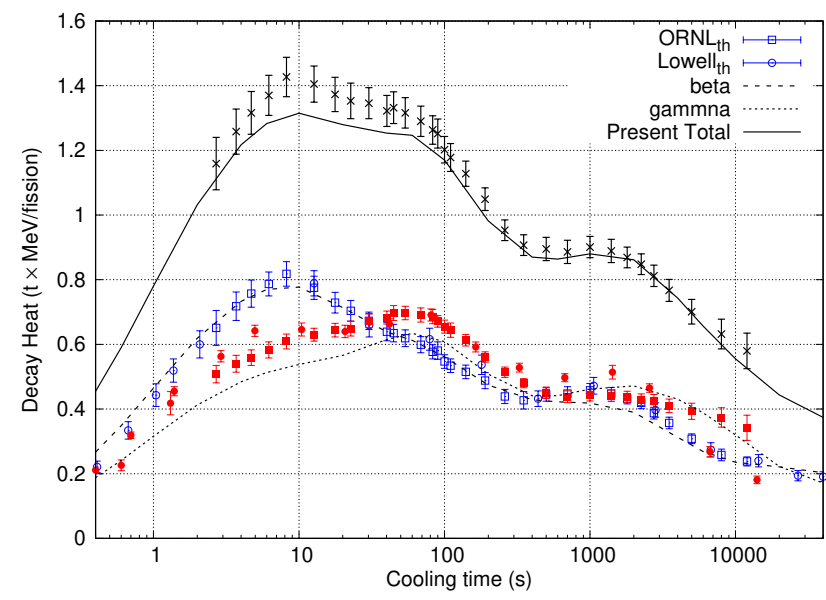

Figure 5. Decay heat as a function of the cooling time (s) calculated from the independent FPY by the summation calculation method. The experimental data of ${ }^{235} U+$ thermal neutron are shown for comparison. Open symbols are decay heat released from $\beta$, filled symbols are that from $\gamma$, cross symbols represent the total energy release.

Hauser-Feshbach statistical decay model and the resulted independent FPY was subsequently used as input of $\beta$ decay calculation. This study showed the feasibility of using theoretically calculated distributions and their influence on fission observables, such as the prompt neutron multiplicity $\bar{v}$, independent FPY $Y_{I}(Z, A, M)$, decay heat, and delayed neutron yield $v_{d}$.

We utilize the primary fission fragment mass yield $Y_{P}(A)$, the fragment mass averaged TKE $\langle\mathrm{TKE}\rangle(A)$ and the width of TKE $\sigma_{\mathrm{TKE}}(A)$ of ${ }^{235} \mathrm{U}$ from low energy fission simulation $[7,8]$. These distributions are used as input of the Hauser-Feshbach statistical decay model [17]. The calculated $\bar{v}(A)$ disagreed with experimental data in the symmetric region $(105 \leq A \leq 130)$. Particularly, we found that $Y_{P}(A)$ and $\langle\mathrm{TKE}\rangle(A)$ of symmetric region $(105 \leq A \leq$ 130) are influential in $\bar{v}$. As a result, the independent FPY $Y_{I}(A)$ also showed disagreement with ENDF/B-VII evaluation. The isotope distributions of cumulative FPY $Y_{C}(Z, A, M)$ of some selected elements around peak region showed good agreement with the evaluated FPY data. The calculated decay heat from $\beta$ decay showed slight disagreement in the early decay time $(\leq 100 \mathrm{~s})$ from the energy release from $\gamma$. The delayed neutron yield $v_{d}$ was 0.01623 which is a few percent larger than the evaluated data. We found that the use of the calculated primary distributions does not yet possess enough precision to apply for the nuclear evaluation, however, this method would be the standard way that allows us to evaluate the fission observables with correlation and consistency when the theoretical prediction reaches the adequate level.

\section{Acknowledgments}

SO would like to thank C.Ishizuka and M.D.Usang to share the calculated data by Langevin model, and
T.Yoshida for helpful discussions. TK carried out this work under the auspices of the National Nuclear Security Administration of the U.S. Department of Energy at Los Alamos National Laboratory under Contract No. 89233218CNA000001.

\section{References}

[1] N. Schunck, L.M. Robledo, Reports on Progress in Physics 79, 116301 (2016)

[2] S. Lemaire, P. Talou, T. Kawano, M.B. Chadwick, D.G. Madland, Phys. Rev. C 72, 024601 (2005)

[3] B. Becker, P. Talou, T. Kawano, Y. Danon, I. Stetcu, Phys. Rev. C 87, 014617 (2013)

[4] J. Randrup, P. Möller, Phys. Rev. Lett. 106, 132503 (2011)

[5] J. Randrup, P. Möller, A.J. Sierk, Phys. Rev. C 84, 034613 (2011)

[6] A.J. Sierk, Phys. Rev. C 96, 034603 (2017)

[7] M.D. Usang, F.A. Ivanyuk, C. Ishizuka, S. Chiba, Phys. Rev. C 94, 044602 (2016)

[8] C. Ishizuka, M.D. Usang, F.A. Ivanyuk, J.A. Maruhn, K. Nishio, S. Chiba, Phys. Rev. C 96, 064616 (2017)

[9] S. Lemaire, P. Talou, T. Kawano, M.B. Chadwick, D.G. Madland, Phys. Rev. C 73, 014602 (2006)

[10] J. Randrup, R. Vogt, Phys. Rev. C 80, 024601 (2009)

[11] P. Talou, AIP Conference Proceedings 1175, 261 (2009)

[12] O. Litaize, O. Serot, Phys. Rev. C 82, 054616 (2010)

[13] T. Kawano, P. Talou, M.B. Chadwick, T. Watanabe, Journal of Nuclear Science and Technology 47, 462 (2010)

[14] O. Litaize, O. Serot, L. Berge, The European Physical Journal A 51, 177 (2015)

[15] K. Oyamatsu, H. Takeuchi, M. Sagisaka, J. Katakura, Journal of Nuclear Science and Technology 38, 477 (2001)

[16] T. Kawano, P. Talou, I. Stetcu, M. Chadwick, Nuclear Physics A 913, 51 (2013)

[17] S. Okumura, T. Kawano, P. Jaffke, P. Talou, S. Chiba, Journal of Nuclear Science and Technology 55, 1009 (2018)

[18] S. Okumura, T. Kawano, P. Jaffke, P. Talou, T. Yoshida, S. Chiba, CERN Proc. 1, 173 (2019)

[19] A.C. Wahl, Atomic Data and Nuclear Data Tables 39, 1 (1988)

[20] T. Ohsawa, T. Horiguchi, H. Hayashi, Nuclear Physics A 653, 17 (1999)

[21] P. Talou, B. Becker, T. Kawano, M.B. Chadwick, Y. Danon, Phys. Rev. C 83, 064612 (2011)

[22] P. Jaffke, P. Möller, P. Talou, A.J. Sierk, Phys. Rev. C 97, 034608 (2018)

[23] T. Kawano, M.B. Chadwick, Journal of Nuclear Science and Technology 50, 1034 (2013)

[24] S. Okumura, T. Kawano, S. Chiba, EPJ Web Conf. 211, 04005 (2019) 